\title{
Dos géneros nuevos de caracoles terrestres (Stylommatophora: Arionacea) de Chile
}

\author{
Patricio Vargas-Almonacid ${ }^{1} \&$ José R. Stuardo $^{2 *}$ \\ 1 Departamento de Ciencias del Desarrollo, Programa de Educación e Investigación Biológica \& Ambiental (Programa- \\ IBAM), Universidad de Los Lagos, Coyhaique-Chile, Teléfono (56-067) 235588, Fax 238777; pvargas@ulagos.cl \\ 2 Departamento de Oceanografía, Universidad de Concepción, Concepción Chile. \\ * $\quad$ Articulo financiado por la Dirección de Investigación Universidad de Concepción (Proyecto DIUC-201.113.059- \\ $1.0)$.
}

Recibido 12-VI-2002. Corregido 04-IX-2006. Aceptado 15-XII-2006.

\begin{abstract}
Two new genera of land snails (Stylommatophora: Arionacea) from Chile. We describe two new genera of land molluscs found under wet leaf litter in isolated fragments of a secondary native forest at the Hualpén Botanical Park $\left(36^{\circ} 45^{\prime}-36^{\circ} 49^{\prime}\right.$ S, $73^{\circ} 9^{\prime}-73^{\circ} 13^{\prime}$ W), University of Concepción, Chile. The new taxa are Pichikadi gen.n., of Punctidae, and Chellius gen.n., of Charopidae, with the following new species: Pichikadi hualpensis sp.n., and Chellius piramidalis sp.n. English diagnosis are presented for all taxa, together with an English identification key and English versions of figure captions and table headings. To facilitate subsequent studies and interpretations, we follow recent authors in using mainly shell characters, an approach that has favored the present taxonomic stability of the world's land micromollusca. The diagnosis and observations are complemented with a key for the species of these families inhabiting continental Chile. Rev. Biol. Trop. 55 (2): 693-708. Epub 2007 June, 29.
\end{abstract}

Key words: Charopidae, land snails, Punctidae, Pulmonata, Micromollusca, Stylommatophora.

La importante diversidad que se está conociendo actualmente en los táxones de caracoles terrestres, particularmente en los micromoluscos endodontoides o endodontoídeos (sensu Solem 1976), no fue reconocida con anterioridad debido a que el grupo permaneció virtualmente desconocido hasta la primera mitad del siglo XX. El $76 \%$ de las especies en el ámbito mundial fueron descritas con anterioridad a 1890 (Solem 1976), aparte de algunos bosquejos radulares publicados entre los años 1870 y 1890 , y las disecciones de tres especies del género Endodonta de Hawaii (Cooke 1928), no existían otros antecedentes anatómicos en la literatura especializada. Debido a su pequeño tamaño, no presentaban interés económico, y el escaso desarrollo de la ecología, no permitía entender su diversidad, la que sólo podía estimarse contando las especies dispersas en las grandes colecciones de los museos del mundo. En la actualidad, sólo el concepto de ecosistema ha permitido reconocer relaciones directas de los micromoluscos con la estructura y desarrollo de los bosques nativos (Stanisic 1990).

Los micromoluscos son organismos pequeños de los que un $96.4 \%$ son menores de $7 \mathrm{~mm}$ en su tamaño máximo (Solem 1976). En su mayoría son habitantes inconspicuos, secretos de la hojarasca, encontrados en suelos cubiertos por árboles caídos, bosques nativos densos y diversos hábitats asociados (Solem et al. 1981, Solem y Climo 1985, Stuardo y Vega 1985, Valdovinos y Stuardo 1989, Stanisic 1990, Vargas-Almonacid 2000). Se encuentran casi siempre muertos, lo que determina que su sistemática y taxonomía se base en primera instancia en estudios conquiológicos. 
En Chile, estudios y revisiones acerca de moluscos endodontoides son escasos, destacándose una primera sinopsis evaluadora del estado taxonómico de las especies descritas para el país, su distribución y posible origen (Stuardo y Vega 1985); la descripción de una nueva especie del género Austrodiscus (Valdovinos y Stuardo 1989), de discutida clasificación por Fonseca y Thomé (1993) y una nueva especie del género Radiodiscus perteneciente a la familia Charopidae (Vargas-Almonacid 2000). Recientemente, se han iniciado investigaciones sobre los complejos problemas de sinonimia y taxonomía, en general de las especies de moluscos terrestres de Chile (Stuardo y VargasAlmonacid 2000, Vargas-Almonacid 2002) y en particular del nuevo ordenamiento de los micromoluscos terrestres chilenos (VargasAlmonacid y Stuardo, en prensa).

Frente a esta realidad y a la gran diversidad de técnicas y táxones utilizados para la evaluación de la biodiversidad en bosques templados, sorprende el escaso interés prestado a los invertebrados y en particular a los moluscos terrestres. No se han utilizado como indicadores de biodiversidad debido principalmente a que no se cree o se sabe que existan en el bosque y que sean tan diversos (Lydeard et al. 2004). En este sentido, el hallazgo de nuevas y diversas categorías taxonómicas está implícito el aportar nuevos e importantes antecedentes para entender el endemismo, la filogenia y la zoogeografía del grupo en la posible relación de la fauna de los moluscos terrestres chilenos con las vertientes Gondwánicas y Pacíficooccidentales (Stuardo y Vega 1985, VargasAlmonacid 2000, 2002).

De la interesante fauna encontrada en el hábitat en estudio y su ecología, en el presente trabajo se describen dos nuevos géneros y dos nuevas especies de micromoluscos terrestres para Chile central.

\section{MATERIALES Y MÉTODOS}

Área de estudio (Fig. 1): El área de estudio forma parte del Parque Botánico de la Universidad de Concepción ubicada en la Península de Hualpén, VIII Región, Chile $\left(36^{\circ} 45^{\prime}-36^{\circ} 49^{\prime} \mathrm{S}, 73^{\circ} 9^{\prime}-73^{\circ} 13^{\prime} \mathrm{W}\right)$.

La reserva está constituida por un paisaje con fragmentos aislados de bosque nativo secundario tipo mosaico, resultado de la alteración y perturbación producida por intervenciones recurrentes de origen antrópico. Esto ha causado cambios en los gradientes naturales, que reflejan un alto grado de fragmentación (Polyméris 1995); sin embargo, esta reserva presenta elementos florísticos de las zonas climatológicas mesomórfica e higromórfica, que corresponden a una vegetación de tipo "parque"; es decir, parches de bosques separados por sectores de vegetación más baja, que de acuerdo con Pisano (1956) parecen constituir una zona vegetacional de transición entre las zonas norte y sur del país.

Muestreo de la malacofauna: El material examinado fue recolectado durante los meses de Agosto a Febrero (2001-2002) en forma aleatoria bajo la hojarasca de seis parches de diferente tamaño (Fig. 1). Cada fragmento fue georeferenciado mediante un navegador personal Garmin GPS45 y la digitalización se basó en fotografías aéreas (1:20 000) utilizando los programas ARC-VIEW 3.2 y ARC INFO NT. Independiente del tamaño del fragmento en estudio y con el objeto de hacer comparables los sitios de muestreo para posteriores análisis ecológicos de los táxones encontrados (VargasAlmonacid et al., en prensa), la superficie total de cada fragmento fue dividida arbitrariamente, en nueve cuadrantes de iguales dimensiones $\left(\mathrm{m}^{2}\right)$ de acuerdo conl tamaño de cada fragmento. En el centro de cada cuadrante se tomó una muestra de hojarasca (1/2 kg balanza manual) sobre una superficie aproximada de $1 \mathrm{~m}^{2}(4.5 \mathrm{~kg}$ por fragmento). Ocasionalmente y debido a que algunos fragmentos presentaban formas irregulares sólo se obtuvo muestras donde existía cubierta vegetacional. La hojarasca así obtenida, fue almacenada en bolsas plásticas, debidamente etiquetadas y cuando fue necesario, fijadas en alcohol al $70 \%$ para su posterior análisis y clasificación. El análisis taxonómico se realizó 


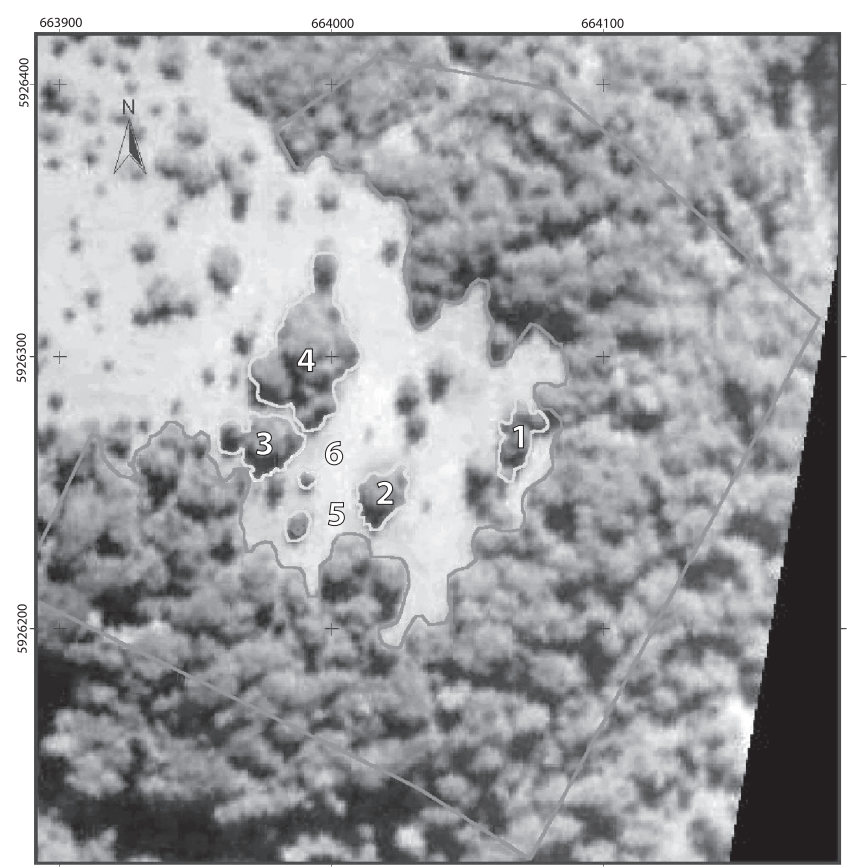

Fig. 1. Área de estudio (fragmentos analizados en blanco); parte del Parque Botánico de la Universidad de Concepción ubicada en la Península de Hualpén, Chile (36 $45^{\prime}-36^{\circ} 49^{\prime}$ S, $\left.73^{\circ} 9^{\prime}-73^{\circ} 13^{\prime} \mathrm{W}\right)$.

Fig. 1. Study area (analyzed fragments in white); part of the Botanical Park of the University of Concepcion at the Peninsula of Hualpén, Chile $\left(36^{\circ} 45^{\prime}-36^{\circ} 49^{\prime} \mathrm{S}, 73^{\circ} 9^{\prime}-73^{\circ} 13^{\prime} \mathrm{W}\right)$.

mediante el estudio morfológico detallado de las conchas (Fig. 2) siguiendo la metodología propuesta por Solem y Climo (1985).

El examen de los detalles finos de las conchas se realizó mediante fotografías al microscopio electrónico de barrido usando un equipo Autoscan U-1 Siemen ETEC (Laboratorio de Microscopía Electrónica de la Universidad de Concepción), utilizando la técnica recomendada por Solem $(1970,1972)$. Junto a la descripción de los caracteres de cada nuevo género y especie como diagnosis de uno u otro, según corresponda, se definen las características de las dos familias y subfamilias que son aquí citadas fehacientemente por primera vez en Chile. Se complementa este estudio con una clave de identificación de las especies encontradas.

\section{RESULTADOS}

El estudio del material obtenido permitió determinar dos géneros nuevos y dos especies nuevas para la ciencia: Pichikadi gen.n., de la familia Punctidae Morse, 1864 y Chellius gen. n., de la familia Charopidae Hutton, 1884, y las respectivas especies Pichikadi hualpensis sp.n., y Chellius piramidalis sp.n.

\section{Familia Punctidae \\ Morse, 1864}

Generalmente pequeños a diminutos endodontoides, primitivamente con una fina escultura apical espiral y una combinación pos-embrional macro y microradial de origen periostracal, 


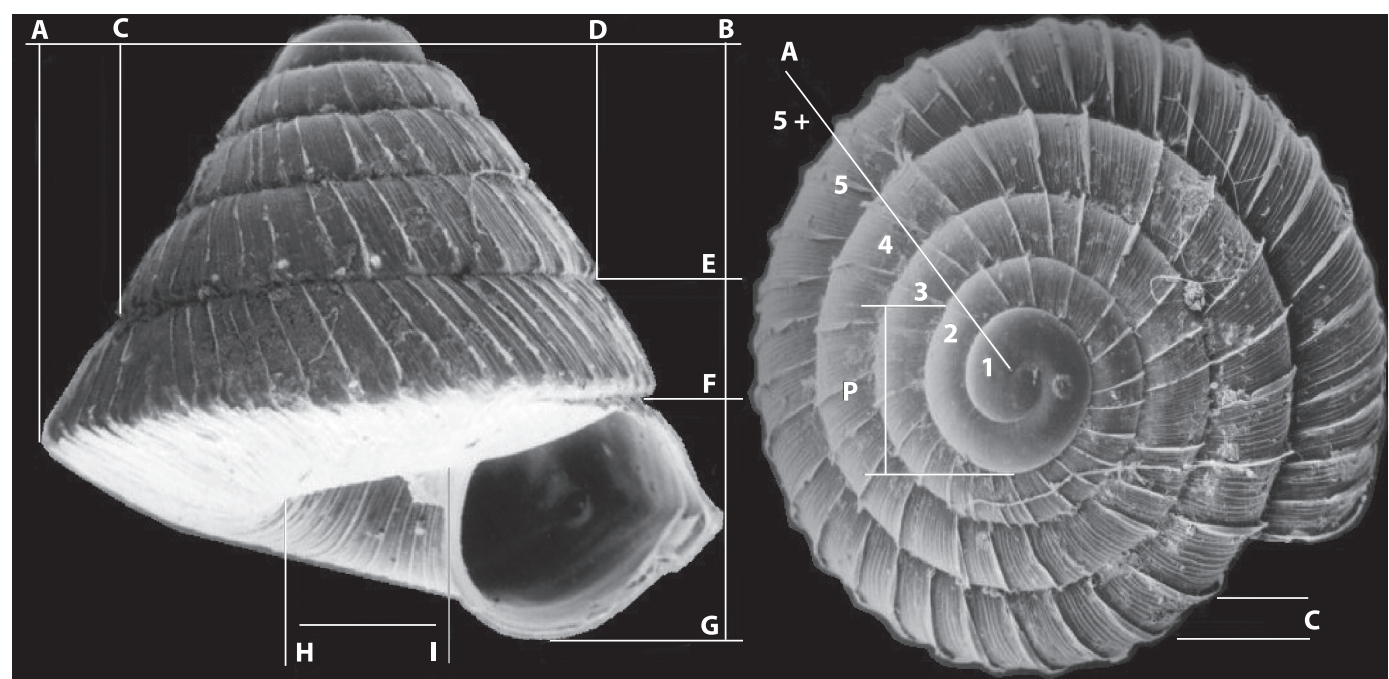

Fig. 2. Medidas básicas de la concha (mm), utilizando los criterios de Solem y Climo (1985): A-B= Diámetro de la concha; $\mathrm{C}-\mathrm{D}=$ Diámetro de la espira; $\mathrm{B}-\mathrm{G}=$ Altura de la concha; $\mathrm{B}-\mathrm{E}=$ Elevación de la espira; E-G= Altura del cuerpo; E-F= Descenso del cuerpo; $\mathrm{F}-\mathrm{G}=$ Altura de la abertura; $\mathrm{H}-\mathrm{I}=$ Diámetro del ombligo; $\mathrm{A}=$ Número de anfractos o vueltas; $\mathrm{P}=$ Número de vueltas de la protoconcha; $\mathrm{C}=$ Número de costillas radiales.

Fig. 2. Basic shell measurements (mm), following Solem and Climo (1985): A-B= Shell diameter; C-D= Spire diameter; $\mathrm{B}-\mathrm{G}=$ Shell height; $\mathrm{B}-\mathrm{E}=$ Spire elevation; $\mathrm{E}-\mathrm{G}=$ Body whorl height; $\mathrm{E}-\mathrm{F}=$ Body whorl descension; F-G= Aperture height; $\mathrm{H}-\mathrm{I}=$ Umbilical width; $\mathrm{A}=$ Whorls number; $\mathrm{P}=$ Embrionic whorls number; $\mathrm{C}=$ Radial ribs number.

esta escultura está alterada en varios táxones. La forma de los anfractos es altamente variable, particularmente en los táxones de Nueva Zelanda. Ombligo abierto, de bastante abierto a casi cerrado. Color monocromo o altamente variable. Algunos táxones desarrollan barreras aperturales, pero la mayoría carecen de ellos. La anatomía de la especie nominal Punctum Morse, 1864, ha sido bien discutida e ilustrada por Baker (1927) y reeditada e impresa por Pilsbry (1948). Antecedentes de la formación conquiolar son presentados por Solem (1976), junto a datos anatómicos de la familia de gran calidad ilustrados en Solem (1983).

Pichikadi gen.n.

(Cuadro 1, Fig. 3. A-F).

Especie Tipo: Pichikadi hualpensis sp.n.

Localidad Tipo: Península de Hualpén, VIII Región, Chile.

Diagnosis: Concha pequeña, frágil, subdiscoidal lenticular, unicoloreada café claro amarillento brillante. Espira convexa poco elevada de crecimiento regular a rápido, con el ápice levemente elevado por sobre la protoconcha. Con 3 7/8 a 4 1/4 anfractos convexos. Sutura profunda y bien marcada que termina en la abertura. La superficie externa está cubierta por una escultura de 48 costillas radiales tenues y la superficie interna (ombligo) por una escultura de 30 costillas radiales tenues, que comienzan en forma irregular a partir del segundo anfracto para distribuirse luego en intervalos regulares. Están separadas por una trama de estrías de crecimientos radiales finos y en número regular, las cuales son cruzadas por un sistema muy denso de finas estrías espirales, sólo detectables al microscopio electrónico. Las estrías espirales abarcan toda la superficie de la concha, pero son más sobresalientes en la zona umbilical. Ombligo estrecho poco profundo, aproximadamente igual a $1 / 4$ del diámetro mayor de la concha (Cuadro 1). Abertura semilunar, con el labio externo afilado, levemente dilatado en el margen columelar y sin callosidades en la unión 

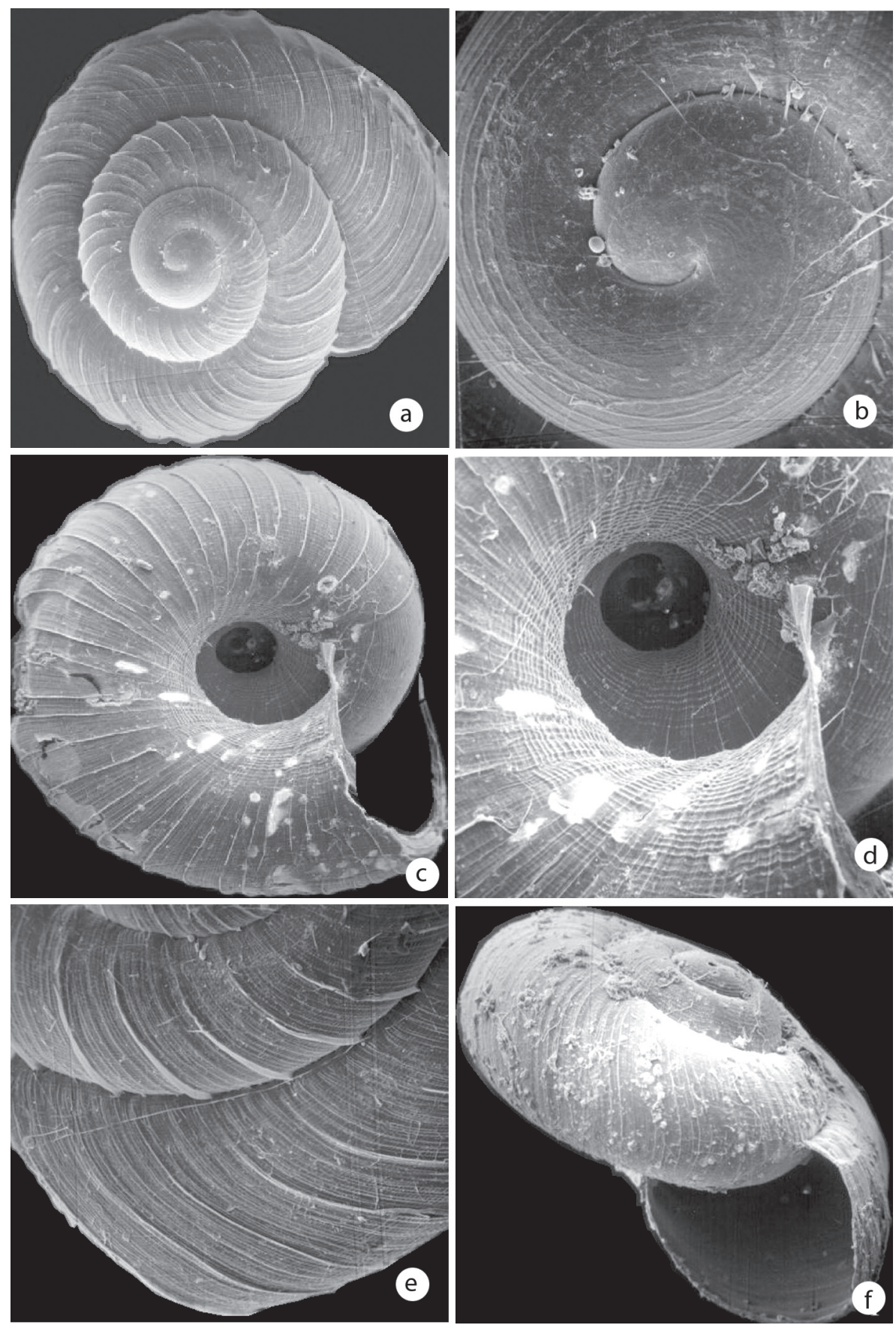

Fig. 3.A. Vista de la superficie dorsal (43x) de un paratipo de P. hualpensis sp.n.; B. Ápice, detalle de la protoconcha, destacando finas cuerdas espirales paralelas (167x); C. Vista general del ombligo (42x); D. Detalle del ombligo (83x); E. Detalle de tercer y cuarto anfractos (84x); F. Vista lateral, mostrando disposición de los anfractos y abertura semilunar (50x).

Fig. 3.A. Dorsal view (43x) of a paratype of P. hualpensis sp.n.; B. Apical sculpture, details of embrionic whorl with parallel spirals cords (167x); C. General view of umbilicus (42x); D. Details of umbilicus (83x); E. Details of third and fourth whorls (84x); F. Lateral view showing whorls position and aperture semilunate (50x). 
CUADRO 1

Medidas de la concha del Holotipo y Paratipos de Pichikadi hualpensis sp. n. (en mm), utilizando los criterios de Solem y Climo (1985)*

TABLE 1

Shell measurements of the Holoype and Patatypes of Pichikadi hualpensis sp. n. (in mm), using the approaches of Solem and Climo (1985)*

$\begin{array}{lllllllllll}\text { Tipo museo/medidas } & \text { A-B } & \text { C-D } & \text { B-G } & \text { B-E } & \text { E-G } & \text { E-F } & \text { F-G } & \text { H-I } & \text { A } & \text { P } \\ \text { Holotipo N } 26799 & 1.75 & 0.85 & 1.02 & 0.12 & 0.90 & 0.17 & 0.73 & 0.36 & 41 / 4 & 2 \\ \text { Paratipo 1= 26800 } & 1.78 & 0.78 & 1.09 & 0.12 & 0.97 & 0.24 & 0.73 & 0.26 & 4 & 2 \\ \text { Paratipo 2=26801 } & 1.60 & 0.78 & 1.02 & 0.17 & 0.85 & 0.19 & 0.65 & 0.26 & 41 / 4 & 2 \\ \text { Paratipo 3=26802 } & 1.48 & 0.65 & 0.92 & 0.09 & 0.82 & 0.17 & 0.65 & 0.24 & 4 & 2 \\ \text { Paratipo 4= 26803 } & 1.85 & 0.90 & 1.09 & 0.14 & 0.95 & 0.17 & 0.78 & 0.48 & 4 & 2 \\ \text { Paratipo 5=26804 } & 1.70 & 0.80 & 1.07 & 0.14 & 0.92 & 0.19 & 0.73 & 0.41 & 41 / 2 & 2 \\ \text { Paratipo 6=26805 } & 1.58 & 0.70 & 1.02 & 0.12 & 0.90 & 0.19 & 0.70 & 0.24 & 37 / 8 & 2 \\ \text { Paratipo 7=26806 } & 1.63 & 0.70 & 0.92 & 0.09 & 0.82 & 0.17 & 0.65 & 0.34 & 37 / 8 & 2 \\ \text { Paratipo 8=26807 } & 2.19 & 1.07 & 1.31 & 0.17 & 1.14 & 0.29 & 0.85 & 0.58 & 4 & 2 \\ \text { Promedio } & 1.72 & 0.80 & 1.05 & 0.12 & 0.91 & 0.19 & 0.71 & 0.35 & \end{array}$

* Abreviaciones en Fig. 2. Abbreviations in fig. 2.

parietal. Protoconcha con escultura de cerca de 15 finas cuerdas espirales paralelas, abarcando los dos primeros anfractos y terminando gradualmente para mezclarse con la microescultura hasta formar la escultura definitiva.

Etimología: El nombre de este género se deriva del Mapuche (Mapudungun) Pichi: pequeño y Kadi: costilla.

Type Species: P. hualpensis sp.n.

Type Locality: Hualpén peninsula, Region VIII, Chile.

English diagnosis: Shell small, fragile, lenticular subdiscoidal, uniformly coloured shiny yellowish to light brown. Spire convex, gently tapering with regular rapid growth and the apex slightly elevated above the protoconch. With $37 / 8$ to 4 1/4 convex whorls. External and internal (umbilical) surfaces sculptured with 48 and 30 slender radial ribs appearing irregularly distributed on the second whorl but becoming regular on the last ones. Ribs separated by a mesh of regular, fine growth striae, crossed by a dense system of spiral striae, only detected with SEM. The spiral striae cover the whole shell surface but become more conspicuous at the umbilical area. Umbilicus narrow, not very deep, approximately equal to a $1 / 4$ of the largest shell diameter (Table 1). Aperture sub-ovate (semilunar), with a sharp, slightly swollen on the columellar, without calluses at the parietal union. Protoconch sculptured with about 15 fine parallel spiral striae, covering the first two whorls and gradually mixing with the microsculpture to form the sculpture proper.

Etymology: The genus name is derived from the Mapuche (Mapudungun) words Pichi: small, and Kadi: rib.

Observaciones: Por la forma de la concha y algunas características de la escultura de la teloconcha, Pichikadi gen.n., presenta 
algunas afinidades con los géneros neozelandeses recientes Phrixgnathus Hutton, 1883 y Taguahelix Powell, 1955. Efectivamente, el análisis de las características de la protoconcha y escultura pos-embrional de ambos géneros realizado por Climo y Goulstone (1993), han sugerido que tanto las características del ápice menos prominente, como el tamaño de la protoconcha, la presencia, distribución y número elevado de microestrías espirales de variada constitución de Taguahelix, sumado a los elementos de escultura postembrional y procesos periostracales de pelos y cerdas característicos en Phrixgnathus, son elementos de valor sistemático para diferenciar ambos géneros.

Sin embargo, existen características de la concha de Pichikadi gen.n., que son únicas, principalmente su pequeño tamaño, y una escultura axial de forma irregular a partir del segundo anfracto, que se distribuye luego en intervalos regulares, separadas por un fino reticulado axial-espiral. Estos caracteres, junto a la ausencia de procesos periostracales (pelos y cerdas) y un menor número de anfractos son típicos, y permiten concluir que corresponden a un patrón genérico, con particularidades propias (Cuadro 1).

\section{Pichikadi hualpensis sp.n. \\ (Cuadro 1, Fig. 3. A-F).}

Descripción: Como especie tipo presenta las mismas características del género.

Etimología: Latinización de la localidad de origen: Hualpén.

Material Examinado: Un holotipo y ocho paratipos, depositados en las Colecciones Científicas de la Universidad de Concepción (UCCC), bajo los números 26799 a 26807.

Discusión: Zilchogyra cleliae Weyrauch, 1965 (de Río Grande do Sul, Brasil) y Radiodiscus misionensis Hylton Scout, 1957b (de Misiones, Argentina) son dos especies sudamericanas que aun cuando corresponden a géneros diferentes presentan similitudes en la concha con $P$. hualpensis sp.n. De acuerdo con la descripción original, Z. cleliae, corresponde a un endodontoide con un diámetro máximo de alrededor de $3 \mathrm{~mm}$, protoconcha completamente lisa ( $\sin$ estriación alguna), vueltas pos-embrionarias con estriación radial y/o espiral, con una espira baja que destaca la última vuelta, y ombligo abierto. Estos antecedentes fueron ponderados por Hylton Scott (1970) quien acepta la presencia de ambas especies; Fonseca y Thomé (1993), al contrario los utilizan para considerar a estas dos especies como sinónimos de Radiodiscus pilsbry Hylton Scout, 1957a, ubicando a esta especie dentro de la familia Charopidae, subfamilia Rotadiscinae Baker, 1927. Sin embargo, tal conclusión necesita reevaluarse ya que el estudio de la ultraescultura de la concha en $Z$. cleliae, realizada por Fernández y Morris (1986, Lám. 1, Fig. 1 y 2), demostró que con 200 aumentos, se observan tenues estrías espirales al final del primer anfracto y comienzo del segundo, que luego se acentúan más, comenzando a mostrar la escultura axial. Estos autores encontraron también, que la escultura postembrional de cóstulas eminentemente radiales, es poco prominente $\mathrm{y}$, dispuesta irregularmente con una separación entre las mismas de aproximadamente cuatro veces su ancho. En los espacios intercostales se observan finas estrías espirales algo onduladas, que con menor aumento aparecen como estrías axiales debido a la ondulación de las mismas (Fernández y Morris 1986, Fig. 3).

Tales antecedentes, deberían ser reconsiderados para analizar su clasificación dentro de la familia Charopidae, subfamilia Rotadiscinae, debido principalmente a que el género Radiodiscus Pilsbry y Ferris, 1906, que incluye hasta ahora al mayor número de endodóntidos americanos, se caracteriza por poseer una concha de tamaño reducido (alrededor de $2 \mathrm{~mm}$ ) con espira baja y la última vuelta de mayor tamaño; una protoconcha formada por estrías o cordones espirales regularmente espaciados y en número constante; vueltas pos-embrionales con una densa escultura radial, compuesta de costillas laminares bajas, de trazado sigmoide y estrías radiales y/o espirales; ombligo abierto, variando en torno a $1 / 4$ del diámetro máximo de la concha, y abertura reniforme (Pilsbry 1948, Solem 1977a, b, 1983, Fonseca y Thomé 
1993, Vargas-Almonacid 2000). Finalmente, aunque en la descripción original de $R$. misionensis, se menciona la escultura axial, no existe información sobre la escultura de la protoconcha. Las otras características de la concha no coinciden con la descripción del género nominal Radiodiscus, ni mucho menos con la de $R$. pilsbry. Por último, Fonseca y Thomé (1993), examinaron dos ejemplares de $R$. pilsbry, pero no mencionan haber revisado algún ejemplar de $R$. misionensis.

De acuerdo con estos antecedentes, y a las similitudes conquiológicas con las descripciones originales de estas especies, parece más razonable proponer la transferencia de las especies Z. cleliae y $R$. misionensis, al género Pichikadi gen.n., familia Punctidae, con la correspondiente sinonimia y combinaciones nuevas.

\section{Familia Charopidae}

Hutton, 1884

Pequeños a grandes endodontoides, escultura apical de la concha muy diversa, vueltas pos-embrionales con grandes costillas radiales y una microescultura de costillas radiales y elementos espirales secundarios. Las demás características de la teloconcha están compuestas sólo de elementos periostracales. Muchos táxones con escultura secundaria o ausente. Anfractos y aspecto de la concha muy variables. Ombligo ampliamente abierto o cerrado. Color monocromo o con la presencia de bandas coloreadas de distintos tonos e intensidades. Barreras aperturales desarrolladas en forma independiente en distintos linajes, sin embargo, esto constituye la excepción y no la regla como en la familia Endodontidae. Mayor información sobre la familia Charopidae se puede encontrar en las excelentes revisiones desarrolladas por Solem (1976, 1983).

\section{Subfamilia Charopinae Hutton, 1884}

Especies pequeñas a grandes con escultura apical formada por cuerdas espirales, presencia de costillas radiales o protoconcha secundariamente desnuda con la presencia de extrañas formaciones. Escultura postapical típicamente charopidae o secundariamente reducida.

Una descripción y discusión más detallada de la subfamilia son presentadas por Solem (1983) y Stanisic (1990). Al igual que estos autores, consideramos que indudablemente esta diagnosis puede ser modificada a la luz de nuevos antecedentes que consideren la distribución y presencia de los táxones que poseen distribuciones más generalizadas o limítrofes.

\section{Chellius gen.n.}

(Cuadro 2, Fig. 4. A-F)

Especie Tipo: Chellius piramidalis sp.n.

Localidad Tipo: Península de Hualpén, VIII Región, Chile.

Diagnosis: Concha pequeña, piramidal de color café claro brillante con pequeñas bandas axiales sigmoidales de color café oscuro distribuidas regularmente entre las costillas. Escultura axial radial fuerte sobrepasada por estructuras periostracales en forma de cuchillas. Espira convexa, con angulación basal de crecimiento regular a rápido; base plana, umbilicada. Ápice convexo moderadamente elevado por sobre la protoconcha. Concha con $51 / 2$ a $61 / 8$ anfractos, que se desarrollan en forma ascendente. Superficies externa e interna (ombligo) cubiertas por 213 y 167 costillas radiales, respectivamente, gruesas, levemente sigmoidales que presentan en su base crestas en forma de cuchillas con una inclinación de 45 grados. Costillas separadas en intervalos regulares por una microescultura de crecimiento formada de 4-11 cóstulas radiales secundarias finas y regularmente espaciadas (Cuadro 2). No se observa estriación espiral. Ombligo estrecho y profundo de aproximadamente 1/4 del diámetro mayor de la concha, aparentemente equivalente a la cámara de fecundación de otras especies. Abertura más ancha que alta, diagonal y sin callosidades en la unión parietal, con peristoma afilado y aquillado, que recuerda a un sifonostoma. Protoconcha lisa, regular, de dos vueltas que terminan abruptamente con la escultura definitiva. 

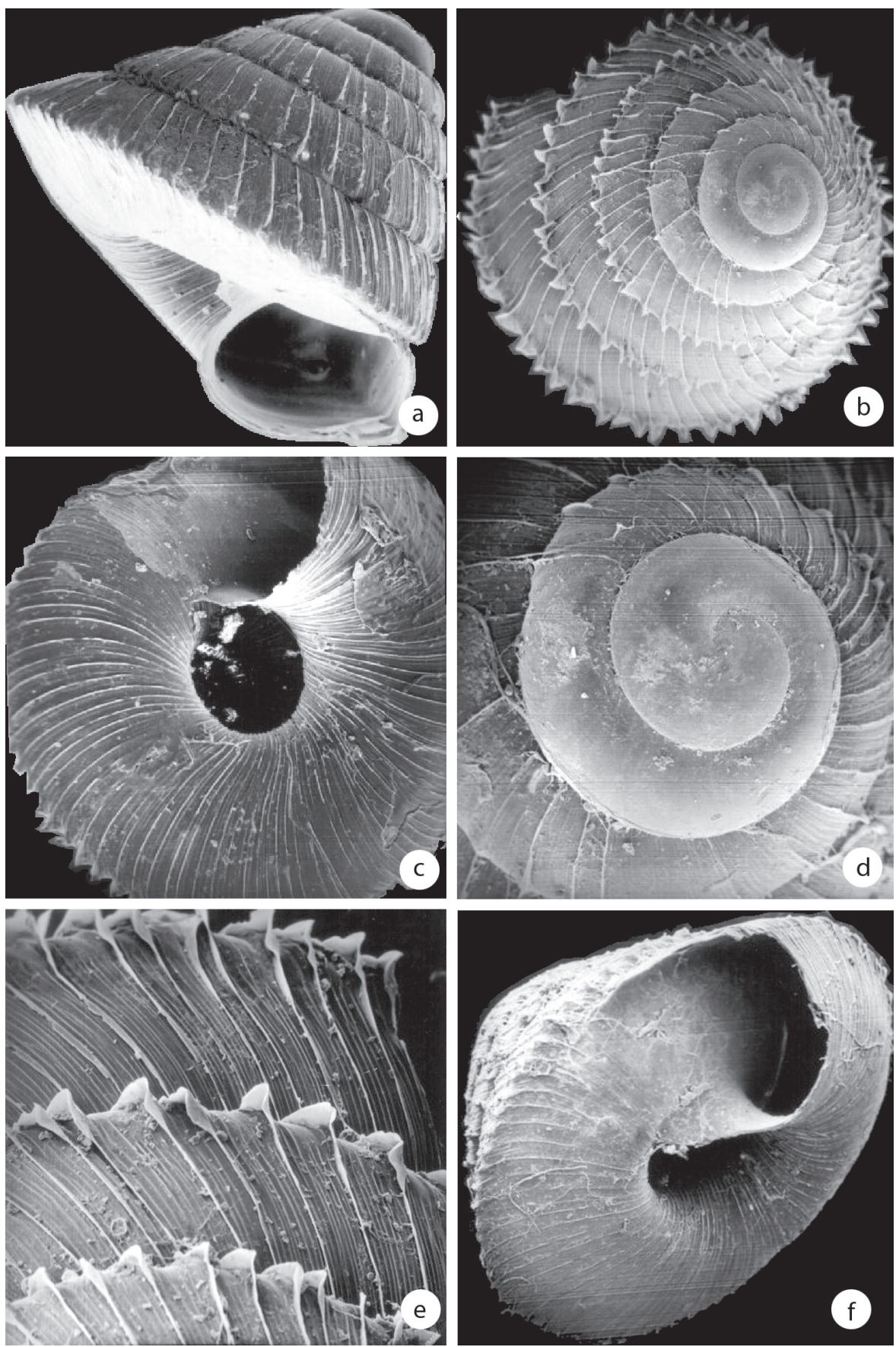

Fig. 4.A. Vista lateral de un paratipo de C. piramidalis sp.n. (20x); B. Vista dorsal (24x); C. Detalle del ombligo (25,5x); D. Escultura apical, detalle de la protoconcha lisa (60x); E. Detalle de los anfractos 5 y 6 (60x); F. Vista ventral, destacando el ombligo estrecho (21x).

Fig. 4.A. Lateral view of a paratype of C. piramidalis sp.n. (20x); B. Dorsal view (24x); C. Details of the umbilicus (22,5x); D. Apical sculpture; details of the smooth whorls (60x); E. Details of whorls five and six (60x); F. Ventral view; details of the narrow umbilicus $(21 \mathrm{x})$. 
CUADRO 2

Medidas de la concha del Holotipo y Paratipos de Chellius piramidalis sp. n. (en mm), según los criterios de Solem y Climo (1985)

TABLE 2

Shell measurements of the Holotype and Paratypes of Chellius piramidalis sp. $n$. (in mm), following Solem and Climo (1985)

\begin{tabular}{|c|c|c|c|c|c|c|c|c|c|c|c|}
\hline Tipo museo/medidas & A-B & C-D & B-G & B-E & E-G & E-F & F-G & H-I & A & $\mathrm{P}$ & $\mathrm{C}$ \\
\hline Holotipo $\mathrm{N}^{\mathrm{o}} 26777$ & 3.26 & 2.34 & 2.73 & 1.17 & 1.56 & 0.58 & 0.97 & 0.63 & $51 / 2$ & 2 & 175 \\
\hline Paratipo $1=26778$ & 3.51 & 2.34 & 2.92 & 1.17 & 1.75 & 0.53 & 1.21 & 0.82 & $514 / 16$ & 2 & 163 \\
\hline Paratipo $2=26779$ & 3.17 & 2.34 & 2.68 & 1.17 & 1.51 & 0.53 & 0.97 & 0.78 & $51 / 2$ & 2 & 172 \\
\hline Paratipo $3=26780$ & 3.51 & 2.43 & 2.97 & 1.21 & 1.75 & 0.63 & 1.12 & 0.73 & $51 / 8$ & 2 & 188 \\
\hline Paratipo $4=26781$ & 3.41 & 2.24 & 2.58 & 1.07 & 1.51 & 0.53 & 0.97 & 0.78 & $51 / 8$ & 2 & 173 \\
\hline Paratipo $5=26782$ & 3.41 & 2.48 & 2.82 & 1.31 & 1.51 & 0.53 & 0.97 & 0.87 & $51 / 8$ & 2 & 201 \\
\hline Paratipo $6=26783$ & 3.41 & 2.24 & 2.48 & 0.97 & 1.51 & 0.53 & 0.97 & 0.78 & $52 / 8$ & 2 & 199 \\
\hline Paratipo $7=26784$ & 3.26 & 2.19 & 2.58 & 1.12 & 1.46 & 0.53 & 0.97 & 0.73 & $61 / 8$ & 2 & 191 \\
\hline Paratipo $8=26785$ & 3.21 & 2.19 & 2.48 & 1.17 & 1.31 & 0.48 & 0.82 & 0.78 & $52 / 8$ & 2 & 194 \\
\hline Paratipo $9=26786$ & 3.02 & 2.19 & 2.53 & 1.02 & 1.51 & 0.53 & 0.97 & 0.68 & $51 / 2$ & 2 & 167 \\
\hline Paratipo $10=26787$ & 3.70 & 2.48 & 3.12 & 1.31 & 1.80 & 0.58 & 1.21 & 1.17 & $51 / 2$ & 2 & 213 \\
\hline Promedio & 3.35 & 2.31 & 2.71 & 1.15 & 1.56 & 0.54 & 1.01 & 0.79 & & & 185.09 \\
\hline Máximo & 3.70 & 2.48 & 3.12 & 1.31 & 1.80 & 0.63 & 1.21 & 1.17 & & & 213 \\
\hline Mínimo & 3.02 & 2.19 & 2.48 & 0.97 & 1.31 & 0.48 & 0.82 & 0.63 & & & 163 \\
\hline Des. Estándar & 0.19 & 0.11 & 0.21 & 0.10 & 0.14 & 0.03 & 0.11 & 0.14 & & & 16.07 \\
\hline
\end{tabular}

Etimología: Nombre derivado del Mapuche (Mapudungun) Chelliu: caracol.

Type Species: $C$. piramidalis sp.n.

Type Locality: Hualpén peninsula, Region VIII, Chile

English diagnosis: Shell small, pyramidal, shiny light brown coloured with small axial bands of dark brown color regularly distributed among the ribs. Axial sculpture strong, projected by periostracal knife-like structures. Spire convex, with a basal angle of growth regular to rapid, base flat, umbilicated. Apex convex, moderately elevated above the protoconch. Shell with $51 / 2$ to $61 / 8$ whorls gradually developed in ascendant way. External and internal (umbilical) surfaces covered by 213 and 167 thick, slightly sigmoidal radial ribs, respectively, with basis presenting knife-like crests with an inclination of $45^{\circ}$. Ribs separated at regular intervals by a growth microsculpture formed by 4-11 fine, regularly spaced, secondary radial costellae (Table 2). Umbilicus narrow, deep, approximately equal to $1 / 4$ of the largest shell diameter; apparently equivalent to the fecundation chamber of other species. Aperture wider than higher, diagonal, without callosities in the parietal union; peristoma sharp, keel-like resembling a siphostoma. Protoconch smooth, regular, with two whorls abruptly ending where the sculpture begins.

Etymology: The genus name is derived from the Mapuche (Mapudungun) word Chelliu: snail.

Observaciones: La concha de Chellius gen.n., presenta un leve parecido con los géneros neozelandeses recientes Aeschrodomus Pilsbry, 1892 y Pulchridomus Climo, 1980; sin embargo, poseen forma turriteloide o de 
domo y extrañas formaciones periostracales en forma de agujas, crestas y pelos o setas de gran variación. Pero, no hay en estos géneros, especies con forma piramidal con estructuras periostracales en forma de cuchillas y un peristoma aquillado.

Chellius gen.n., tampoco presenta afinidades con los géneros sudamericanos hasta ahora descritos, salvo similitud en la escultura lisa de la protoconcha, carácter presentado también por los géneros: Zilchogyra Weyrauch, 1965, Trochogyra Weyrauch, 1965, y Stephanoda Albers, 1860. Sin embargo, éstos presentan otras características en la forma y altura de la concha, abertura, ombligo, y otras diferencias constatables en las descripciones originales y comentarios de Hylton Scott (1970), Fernández y Morris (1986), y Fonseca y Thomé (1993).

\section{Chellius piramidalis sp.n. \\ (Cuadro 2, Fig. 4. A-F)}

Descripción: Especie tipo con las mismas características genéricas.

Etimología: Hace referencia a la forma piramidal de la concha.

Material Examinado: Un holotipo y diez paratipos, depositados en las Colecciones
Científicas de la Universidad de Concepción (UCCC), bajo los números 26777 a 26787.

Observaciones: Según Solem (1976), las extrañas formaciones periostracales de los Charopidae (como son las cuchillas existentes en cada una de las costillas mayores), se observan generalmente en especímenes que habitan en ambientes ricos en limo y carbonato de calcio. Por otra parte, un ombligo estrecho y profundo, corresponde a un carácter constante presente en los Endodontidae de las islas del Pacífico, aunque según Solem (1976) se ha observado en un sólo Charopidae Tuimalida infundibulus (Hombron y Jacquinot 1841).

Se ha descrito que este ombligo estrecho y profundo es utilizado como cámara de fecundación donde se albergan cápsulas de huevos (Climo 1970, Solem 1976, Climo 1980) sirviendo eventualmente como refugio de arañas e insectos. Sin embargo, no es posible aun resolver si la presencia de este patrón en especies de Nueva Zelanda tales como Fectocola marsupialis Powell, 1941 o Aeschrodomus worleyi Powell, 1928 (Climo 1970, 1980) y en C. piramidalis sp.n. de Chile, sería también una respuesta que corresponde a factores de estabilidad, baja predación y escaso impacto humano, como propone Solem (1983) o si corresponde a otros patrones adaptativos.

\section{Clave de identificación de las especies descritas}

La siguiente clave considera las medidas conquiológicas estándar (Fig. 2) propuestas por Solem y Climo (1985), diseñada sólo para permitir la identificación de especímenes adultos. Se incluyen las especies sudamericanas consideradas en los comentarios.

1a. Escultura de la protoconcha con estrías finas o pequeñas cuerdas espirales; costillas radiales delgadas, poco prominentes, cruzadas por finas estrías espirales que forman un reticulado denso; concha subdiscoidal lenticular .

1b. Escultura de la protoconcha desnuda; costillas radiales gruesas, sin estriación espiral; concha de forma piramidal; peristoma afilado y aquillado, que recuerda a un sifonostoma . . . . . . . . . . . Chellius piramidalis sp.n.

2a. Concha pequeña de consistencia frágil; con periostraco fino de coloración más o menos brillante . . . . . . . . . (3)

2b. Concha pequeña gruesa con periostraco tosco, opaco . . . . . . . . . Radiodiscus misionensis Hylton Scott, 1957a

3a. Estriación axial superior con aproximadamente 48 costillas radiales tenues; ombligo estrecho poco profundo aprox. 1/5 del diámetro mayor de la concha; abertura semilunar; labio externo afilado, dilatado en el margen columelar

Pichikadi hualpensis sp.n.

3b. Estriación axial superior con aproximadamente 33 costillas radiales tenues; ombligo estrecho poco profundo aprox. 1/4 del diámetro mayor de la concha; abertura redondeada; labio externo afilado muy frágil no dilatado en el margen

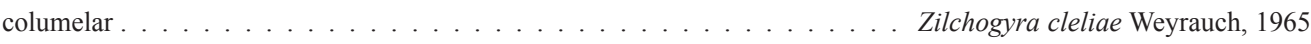




\section{Key to identify the described species}

The following key employs the standard shell measurements (Fig. 2) proposed by Solem and Climo (1985), for the identification of adult specimens. The South American species mentioned in the remarks are also included.

1a. Protoconch sculpture with fine striae or small spiral cords; radial ribs thin, little prominent, crossed by fine spiral striae forming a dense reticulum; shell lenticular subdiscoidal . . . . . . . . . . . . . . . . . . . . (2)

1b. Protoconch sculpture nude, or with difuse radial wrinkles; radial ribs thick, without spiral striation; pyramidal shell; peristome sharp, keel-like, resembling a sophonostoma . . . . . . . . . . Chellius piramidalis sp.n.

2a. Shell small, fragile; priostracum thin with more or less lustrous coloration . . . . . . . . . . . . . . . . (3)

2b. Shell small, thick with opaque, coarse periostracum . . . . . . . . Radiodiscus misionensis Hylton Scott, 1957a

3a. Upper axial striation with about 48 thin radial costellae; umbilicus narrow, not too deep, approximately $1 / 5$ of the greatest shell diameter; aperture semilunar; external lip sharp, swollen at the columellar margin

Pichikadi hualpensis sp.n.

3b. Upper axial striation with about 33 thin radial costellae; umbilicus narrow, not deep, approximately $1 / 4$ of the largest shell diameter; aperture circular; external lip sharp, very fragile, not swollen at the columellar margin.

Zilchogira cleliae Weirauch, 1965

\section{DISCUSIÓN}

Desde el punto de vista taxonómico, debemos destacar, que los estudios conquiológicos son bastante peculiares entre los endodontoides, permitiendo la diferenciación y clasificación entre familias (Solem 1976, 1983, Stanisic 1990, Fonseca y Thomé 1993, Vargas-Almonacid 2000), reconociéndose actualmente que algunas características de la concha, incluyendo escultura apical, microescultura embrional y postembrional, muestran correlaciones positivas con patrones anatómicos fundamentales (Stanisic 1990, Schander y Sundberg 2001).

Por otro lado, y a pesar de hacer algunas consideraciones zoogeográficas preferimos no especular acerca del posible origen faunístico de los micromoluscos terrestres chilenos, atendiendo a que están siendo recientemente estudiado (Vargas-Almonacid 2000, Stuardo y Vargas-Almonacid 2000, Vargas-Almonacid y Stuardo, en prensa). Razón por la cuál, sólo se comentan las relaciones entre los micromoluscos terrestres de las diferentes zonas geográficas estudiadas (eg. Australia, Nueva Zelanda, Islas del Pacifico Occidental), considerando las posibles relaciones Gondwánicas (Solem 1976, 1983, Stuardo y Vega 1985, Climo 1989,
Stanisic 1990) y Pacífico Occidentales (Solem 1983, Stuardo y Vega 1985, Vargas-Almonacid 2000) de las distribuciones actuales de los táxones más representativos.

De acuerdo con estos antecedentes, las unidades taxonómicas mencionadas en este trabajo hacen referencia a su posición sistemática actual, considerándose en términos estrictos las familias Punctidae y Charopidae.

La unidad de familiar Punctidae, es tratada aquí en el sentido estricto propuesto por Solem (1983), incluyendo a la subfamilia Punctinae del Hemisferio Norte (Pilsbry 1948, Burch y Pearce 1990, Forsyth 1999) y no como sugiere Climo (1969a, b) a la superfamilia Punctoidea, raíz de los endodontoides.

La familia Punctidae, es abundante en el este y sur de Australia, Nueva Zelanda, parte de la región Holártica y África (Verdcourt 1972, van Bruggen 1978). Solem (1983) menciona un grupo marginal presente en Polinesia y atribuye la presencia de las especies descritas para Chile por Odhner (1922) en las Islas Juan Fernández (Chile insular) como el producto de una modesta radiación.

La mayoría de las especies de la familia presentes en las Islas del Pacífico Occidental corresponden al género Punctum, taxon de 
origen Holártico (Solem 1983), siendo generalmente asignado a una gran variedad de especies de difícil descripción conquiológica y carencia en general de partes blandas.

De acuerdo con los resultados, en Chile, la familia estaría representada por $P$. hualpensis sp.n., y las especies del género Punctum, $P$. conicum Odhner, 1922 y P. depressum Odhner, 1922 (Odhner 1922, Stuardo y Vega 1985), presentes en la Isla Robinson Crusoe, destacando que son necesarios estudios acerca del estado de conservación actual de estas dos últimas especies debido a la gran presión antrópica sobre estas islas.

En cuanto a la familia Charopidae, podemos decir que, sería un grupo más evolucionado que los Endodontidae, ya sea en estructura y complejidad genital, escultura apical y protoconcha muy diversa, como también en el grado de adaptación y distribución a una mayor diversidad de hábitats (Solem et al. 1981, Solem 1983) y que en términos de especies sería el grupo dominante en relación con los moluscos terrestres de Nueva Zelanda (Climo 1969a, b, 1970, 1980, 1985, Powell 1976, Solem et al. 1981, Solem y Climo 1985, Climo y Goulstone 1993), Nueva Caledonia (Franc 1957, Solem 1961) y diversas partes de Australia (Burch 1976, Solem 1983, Stanisic 1990).

En Norteamérica, estaría bien representada en Canadá, EEUU y México (Pilsbry 1948, Burch y Pearce 1990, Forsyth 1999), reconociéndose para América del Sur su presencia en Argentina, Brasil y Chile (Hylton Scott 1948, 1957a, b, 1970, 1981, Fonseca y Thomé 1993, y Vargas-Almonacid 2000, respectivamente), aunque, tanto la sistemática como su taxonomía estarían lejos de presentar estabilidad, verificándose actualmente el comienzo de un arduo proceso de estudio (Fonseca y Thomé 1993, Vargas-Almonacid 2000, Vargas-Almonacid y Stuardo, en prensa, Sergio Miquel com. pers.).

En este sentido, para los charopinianos (charopinine -estructuras que concuerdan con los patrones típicos de la familia Charopidae- sensu Solem 1976) descritos en este trabajo, seguimos la clasificación propuesta por Solem (1983), la cual incluye las subfamilias Otoconchinae Cockerell, 1893, Rotadiscinae, Charopinae, Trukcharopinae y Semperdoninae Solem, 1983.

Para nuestro país reconocemos la presencia de las subfamilias Rotadiscinae (Fonseca y Thomé 1993, Vargas-Almonacid 2000) y en este trabajo la subfamilia Charopinae, considerando a las especies descritas por Odhner (1922) para Juan Fernández y las presentadas en la compilación de Stuardo y Vega (1985), autores que en su clasificación proponían a la superfamilia Endodontacea y familia Endodontidae.

De igual forma, consideramos los argumentos planteados por Solem (1983), Climo (1989) y Stanisic (1990), quienes mencionan que las afinidades entre estas subfamilias aún son inciertas. En este sentido, estos autores proponen que Otoconchinae, de Nueva Zelanda, sería una desviación secundaria de los Charopinae neozelandeses. Desde el punto de vista anatómico los Trukcharopinae de Micronesia serían un derivado típico de las formas CharopaSinployea Pacífico-Occidentales (Solem 1983) y al parecer estas estructuras no serían equivalentes con las australianas o neozelandesas conocidas. Por otro lado, consideramos que los representantes de la subfamilia Rotadiscinae podrían ser relictuales, ya que su anatomía posee aspectos Punctoideos en partes de la ovotestis. La región paleal se reconoce (Solem 1983, Climo 1989, Stanisic 1990) más primitiva que la de los Charopinae; sin embargo, sus delgados genitales terminales con algunos órganos accesorios serían muy diferentes de los de la familia Punctidae y más relacionados con los Charopidae. En este sentido se podría considerar a la subfamilia Rotadiscinae como el "stock" base de la radiación Charopidiana como propone Solem (1983).

Considerando lo anterior, se propone el siguiente plan general de clasificación que para los nuevos taxónes: 
Clase Gastropoda Cuvier, 1797

Subclase Pulmonata Cuvier, 1817

Superorden Stylommatophora Schmidt, 1856 (= Geophila Férussac, 1812)

Orden Sigmurethra Pilsbry, 1900

Suborden Aulacopoda Pilsbry, 1896

Superfamilia Arionacea Baker, 1955

Familia Punctidae Morse, 1864

Subfamilia Punctinae Morse, 1864

Género Pichikadi novo

Familia Charopidae Hutton, 1884

Subfamilia Charopinae Hutton, 1884

Género Chellius novo

\section{AGRADECIMIENTOS}

Agradecemos a John Stanisic (Queensland Museum), Winston Ponder (Australian Museum), por facilitar sus trabajos personales y literatura especializada de sus respectivos países; Sergio Miquel (CONICET, Argentina) por los importantes comentarios previa realización de este trabajo; Maria E. Casanueva (Lab. Acarología, Depto. Zoología, Universidad de Concepción), Andrés Angulo y Tania Olivares (Lab. Lepidopterología, Depto. Zoología, Universidad de Concepción), por sus comentarios críticos, ayuda y apoyo en laboratorio; Patricio Chandía, Milenko Aguilera (Depto. Zoología, Universidad de Concepción), Julio Crespo y Jaime Rau (Depto. Cs. Básicas y Programa de Educación e Investigación Biológica \& Ambiental, Programa-IBAM, Universidad de Los lagos), por sus sugerencias, comentarios y ayuda en terreno; Hugo Moyano, por sus comentarios y consideraciones últimas. Al personal del Laboratorio de Microscopía de la Universidad de Concepción por la ayuda y facilidades prestadas para la fotografía y tratamiento de los ejemplares, también a Minerva Contreras (Secretaria Colecciones Científicas Universidad de Concepción), por el manejo y clasificación de los ejemplares en el museo, al Laboratorio de Geomática Centro Eula-Chile, por el tratamiento de las fotografías aéreas. Por último a la Dirección de Investigación Universidad de Concepción por financiar este artículo como parte del proyecto DIUC - 201.113.059-1.0.

\section{RESUMEN}

El estudio taxonómico y sistemático de ejemplares de moluscos terrestres recolectados bajo hojarasca en fragmentos aislados de bosque nativo secundario, Parque Botánico Hualpén, $\left(36^{\circ} 45^{\prime}-36^{\circ} 49^{\prime} \mathrm{S}, 73^{\circ} 9^{\prime}-73^{\circ} 13^{\prime} \mathrm{W}\right)$, Universidad de Concepción, Chile, permitió describir dos géneros nuevos Pichikadi gen.n. de la familia Punctidae, y Chellius gen.n. de la familia Charopidae, con las siguientes especies nuevas para la ciencia: Pichikadi hualpensis sp.n. y Chellius piramidalis sp.n. Para facilitar interpretaciones y estudios posteriores, se sigue a diversos autores en describir las características de la concha, que como otros caracteres filogenéticos han dado origen a la aparente estabilidad sistemática de los micromoluscos terrestres en el ámbito mundial. Las descripciones y observaciones de cada género y especies se complementan con una clave para las especies de estas familias encontradas en Chile continental.

Palabras clave: Caracoles terrestres, Charopidae, Punctidae, Pulmonata, Micromoluscos, Stylommatophora.

\section{REFERENCIAS}

Baker, H.B. 1927. Minute Mexican Land Snails. P. Acad. Nat. Sci. Phila. 79: 223-246, P1. 15-20.

Burch, J.B. 1976. Outline of classifications of Australian terrestrial molluscs (native and introduced). J. Malac. Soc. Aust. 3: 127-156.

Burch, J.B. \& T.A. Pearce. 1990. Terrestrial Gastropoda, 201-309. In D.L. Dindal (ed.). Soil Biology Guide. Wiley, Nueva York, EEUU.

Climo, F.M. 1969a. Classification of New Zealand Arionacea (Mollusca: Pulmonata). I. The Higher Classification. Rec. Dom. Mus. Wellington 6: 145-158. 
Climo, F.M. 1969b. Classification of New Zealand Arionacea (Mollusca: Pulmonata). II. A revision of Charopa subgenus Ptychodon Ancey, 1888. Rec. Dom. Mus. Wellington 6: 175-258.

Climo, F.M. 1970. Classification of New Zealand Arionacea (Mollusca: Pulmonata). III. A revision of the genera Charopa Albers, 1860 (excluding subgenus Ptychodon Ancey, 1888), Phenacharopa Pilsbry, 1893 and Flammocharopa n. gen. (Endodontidae: Endodontinae). Rec. Dom. Mus. Wellington 6: 285-366.

Climo, F.M. 1980. Classification of New Zealand Arionacea (Mollusca: Pulmonata). VII. The genera Aeschrodomus Pilsbry and Pulchridomus n. gen. (Charopidae). Nat. Mus. New Zeal. Rec. 1: 293-303.

Climo, F.M. 1985. Classification of New Zealand Arionacea (Mollusca: Pulmonata). XI. The new genus Chaeureopa and description of a new species of Paracharopa Climo (Charopidae). New Zeal. J. Zool. 12: 283-296.

Climo, F.M. 1989. The panbiogeography of New Zealand as illuminated by genus Fectola Iredale, 1915 and subfamily Rotadiscinae Pilsbry, 1927 (Mollusca: Pulmonata: Punctoidea: Charopidae). New Zeal. J. Zool. 16: 587-649.

Climo, F.M. \& J.F. Goulstone. 1993. Descriptions and redescriptions of landsnails (Mollusca: Punctidae) in the genera Phrixgnathus and Taguahelix. Rec. Auck. Inst. Mus. 30: 27-47.

Cooke, C.M. 1928. Three Endodonta from Oahu. Bull. Bernice P. Bishop Mus. 47: 13-27.

Fernández, D. \& M.R. Morris. 1986. Presencia de Zilchogyra cleliae Weyrauch, 1965 (MolluscaPulmonata) para la provincia de Santa Fe (Republica Argentina) y aportes al conocimiento de su estructura conquiolar. Notas Mus. de La Plata 21: 23-29.

Fonseca, Á.L. \& J.W. Thomé. 1993. Descrição de Glabrogyra subgen.n., Recaracterização de Austrodiscus twomeyi (Pardiz, 1954) e Reclassificação das especies Sulamericanas dos Gêneros Austradiscus Parodiz, 1957, Radioconus Baker, 1927, Radiodomus Baker, 1930 e Trochogyra Weyrauch, 1965 (Charopidae) e Zilchogyra Weyrauch, 1965 (Helicodiscidae) (Gastropoda, Stylommatophora, Endodontoidea). Iheringia Sér. Zool. Porto Alegre 75: 97-105.

Forsyth, R.G. 1999. Terrestrial gastropods of the Columbia Basin. Columbia Basin projects. Roy. Brit. Columbia Mus. 133 p.
Franc, A. 1957. Mollusques terrestres et fluviatiles de l'Archipiel NéoCalédonien. Mém- Mus. Hist. Nat. Paris, n. S., sér. A, Zool. 200 p.

Hombron, M. \& H. Jacquinot. 1841. Description de quelques Mollusques provenant de la campagne de Ástrolabe et de la Zélée. Ann. Sci. Nat. Zool. 16: 62-64.

Hylton Scott, M.I. 1948. Moluscos del Biotopo de Cerro Colorado. Acta Zool., Lilloana 4: 229-275.

Hylton Scott, M.I. 1957a. Endodóntidos Neotropicales (Moll. Pulm.). I. Neotropica 3: 7-16.

Hylton Scott, M.I. 1957b. Endodóntidos Neotropicales (Moll. Pulm.). I. Neotropica 3: 79-87.

Hylton Scott, M.I. 1970. Endodóntidos de la región Austral américana. Rev. Mus. Argentino Cien. Natur. Bernardino Rivadavia 10: 267-296.

Hylton Scott, M.I. 1981. Referencia al género Stephanoda Albers, 1860, y la creación del género Stephadiscus gen. nov. Neotropica 78: 123-126.

Lydeard, Ch, R.H. Cowie, W.F. Ponder, A.E. Bogan, P. Bouchet, S.T. Clark, K.S. Cummings, T.J. Frest, O Gargominy, D.G. Herbert, R. Hershler, K.E. Perez, B. Roth, M. Seddon, E.E. Strong \& F.G. Thompson. 2004. The Global Decline of Nonmarine Mollusks. Bioscience 54: 321-330.

Odhner, N.H. 1922. Mollusca from Juan Fernandez and Easter Island, p. 219-254. In C. Skottsberg (ed). The Nat. Hist. of Juan Fernández and Easter Island, vol 3, part 2, 22: 219-254.

Pilsbry, H.A. 1948. Land Mollusca of North America (North of Mexico). Monogr. Acad. Nat. Sci. Phila. 2: 521-1113.

Pilsbry, H.A. \& J.H. Ferris. 1906. Mollusca of the Southwestern States. In II. Proc. Acad. Nat. Sci. Philad. 58: 653 p.

Pisano, E. 1956. Esquema de clasificación de las comunidades vegetales de Chile. Agronomia 2: 30-33.

Polyméris, C. 1995. Vegetación actual de la península de Hualpén: clasificación y dinámica. Tesis de Magister, Universidad de Concepción, Concepción, Chile.

Powell, A.W.B. 1976. Shells of New Zealand. ChristchurchWhitcoulls, Auckland, Nueva Zelanda. 154 p. 
Schander, C. \& P. Sundberg. 2001. Useful Characters in Gastropod Phylogeny: Soft Information or Hard Facts? Syst. Biol. 50: 136-141.

Solem, A. 1961. New Caledonian land and fresh-water snails, an annotated check list. Fieldiana Zool. 41: 415-501.

Solem, A. 1970. Malacological applications of scanning electron microscopy. I. Introduction and Shell surface features. The Veliger 12: 394-400.

Solem, A. 1972. Malacological applications of scanning electron microscopy. II. Radular structure and functioning. The Veliger 14: 327-336.

Solem, A. 1976. Endodontoid Land Snails from Pacific Islands (Mollusca: Pulmonata: Sigmurethra). Part 1: Family Endodontidae. Field Mus. Nat. Hist., Chicago, EEUU. 508 p.

Solem, A. 1977a. Radiodiscus hubrichti Branson, 1975. A synonym of Striatura (S.) pugetensis (Dall, 1895) (Pulmonata: Zonitidae). Nautilus 91: 146-148.

Solem,A. 1977b. Shell Microsculpture in Striatura, Punctum, Radiodiscus and Planogyra. Nautilus 91: 149-155.

Solem, A. 1983. Endodontoid Land Snails from Pacific Islands (Mollusca: Pulmonata: Sigmurethra). Part II. Families Punctidae and Charopidae, Zoogeography. Field Mus. Nat. Hist., Chicago, EEUU. 336 p.

Solem, A. \& F.M. Climo. 1985. Structure and habitat correlations of sympatric New Zealand land snails species. Malacologia 26: 1-30.

Solem, A., F.M. Climo \& D.J. Roscoe. 1981. Sympatric species diversity of New Zealand land snails. N. Zealand J. Zool. 8: 453-485.
Stanisic, J. 1990. Systematics and Biogeography of eastern Australian Charopidae (Mollusca, Pulmonata) from subtropical rainforests. Mem. Queensland Mus. 30: $1-241$

Stuardo, J. \& P. Vargas-Almonacid. 2000. Moluscos terrestres de Chile. Sinonimia y problemas relacionados: 1. Familias Veronicellidae, Pupillidae y Achatinellidae (Gastropoda: Pulmonata). Gayana Zool. 64: 171-188.

Stuardo, J. \& R. Vega. 1985. Synopsis of Land Mollusca of Chile. Whith Remarks on Distribution. Stud. Neotr. Fauna Envir. 20: 125-146.

Valdovinos, C. \& J. Stuardo. 1989. Nuevo Gastropodo Humícola de Chile Austrodiscus (Zilchogyra) solemi spec. nov. (Pulmonata: Endodontidae). Bol. Soc. Biol. Concepción, Chile 66: 239-245.

Van Bruggen, A.C. 1978. Land molluscs, p. 877-923. In M.J.A. Werger (ed.). Biogeography and Ecology of Southern Africa. W. Junk, La Haya, Holanda.

Vargas-Almonacid, P. 2000. Nueva especie de microgastrópodo humícola para Chile, Radiodiscus quillajicola spec. nov. (Stylommatophora: Charopidae). Gayana Zool. 64: 61-66.

Vargas-Almonacid, P. 2002. Micromoluscos terrestres asociados a la hojarasca de remanentes boscosos del Parque Botánico Hualpén (VIII Región, Chile). Tesis Magíster, Universidad de Concepción, Concepción, Chile.

Verdcourt, B. 1972. The zoogeography of the non-marine Mollusca of East Africa. J. Conchol. 27: 291-348.

Weyrauch, W.K. 1965. Cinco Nuevos Endodóntidos de Argentina y Perú. Neotropica 11: 105-115. 